\title{
P104 White Coat Hypertension is Associated with Increased Small Vessel Disease in the Brain
}

\author{
Fran Kirkham ${ }^{1}$, E Drazich ${ }^{1}$, A Vundavalli' ${ }^{1}$, Rankin ${ }^{1}$, J Timeyin ${ }^{1}$, E Bunting ${ }^{1}$, K Ali1 ${ }^{1,2}$, C Rajkumar ${ }^{1,2}$ \\ ${ }^{1}$ Brighton and Sussex University College Hospitals Trust, UK \\ ${ }^{2}$ Department of Medicine, Brighton and Sussex Medical School, Brighton, UK
}

\section{ABSTRACT}

Objective: Small vessel disease, measured by brain white matter hyperintensity (WMH), is associated with increased stroke risk and cognitive impairment. This study aimed to explore the relationship between WMH on computerised tomography (CT) and white coat hypertension (WCH) in patients with recent transient ischaemic attack (TIA) or lacunar stroke (LS).

Methods: Ninety-six patients recruited for the ASIST trial (Arterial Stiffness in Lacunar Stroke and TIA) underwent measurement of clinic blood pressure (BP) and ambulatory BP monitoring (APBM) within two weeks of TIA or LS. Patients were grouped by BP phenotypes. Twenty-three patients had normotension (clinic BP $<140 / 90 \mathrm{mmHg}$ and day-time ABPM $<135 / 85 \mathrm{mmHg}$ ) and 25 patients had WCH (clinic BP $>140 / 90 \mathrm{mmHg}$ and day-time ABPM $<135 / 85 \mathrm{mmHg}$ ). CT brain images were scored for WMH using the four-point Fazekas visual rating scale. Patients were grouped into no-mild WMH (scores $0-1$ ) or moderate-severe (scores 2-3) groups. The relationship between BP and WMH was explored with chi-square and logistic regression accounting for known cardiovascular risk factors (age, gender, smoking, diabetes and hyperlipidaemia).

Results: $44 \%$ of WCH patients had moderate-severe WMH compared to $17 \%$ of normotensives $(p=0.047)$. Logistical regression incorporating $\mathrm{WCH}$ as the independent factor and cardiovascular risk factors as independent variables showed WCH to be the only independent significant factor contributing to WMH $(p=0.024)$.

Conclusion: Patients with WCH were more likely to have moderate-severe WMH on CT brain than normotensives. WCH was associated with increased WMH, independent of other cardiovascular risk factors. This study suggests that WCH is associated with increased small vessel disease in the brain and may benefit from treatment.

\begin{tabular}{lccc}
\hline & Normotension $(\boldsymbol{n}=\mathbf{2 3})$ & WCH $(\boldsymbol{n}=\mathbf{2 5})$ & Statistical significance \\
\hline Female $n(\%)$ & $8(35 \%)$ & $7(28 \%)$ & $p=0.613$ \\
Age (years) & $69.7 \pm 11.79$ & $76.52 \pm 8.69$ & $p=0.026$ \\
Ever Smoker $n(\%)$ & $12(52 \%)$ & $18(72 \%)$ & $p=0.156$ \\
Hyperlipidaemia $n(\%)$ & $18(78 \%)$ & $15(60 \%)$ & $p=0.458$ \\
Diabetes $n(\%)$ & $4(17 \%)$ & $7(28 \%)$ & $p=0.173$ \\
No-Mild WMH $n(\%)$ & $19(83 \%)$ & $14(66 \%)$ & $p=0.047$ \\
Moderate-Severe WMH $n(\%)$ & $4(17 \%)$ & $11(44 \%)$ & $p=0.047$ \\
\hline
\end{tabular}

(c) 2019 Association for Research into Arterial Structure and Physiology. Publishing services by Atlantis Press International B.V. This is an open access article distributed under the CC BY-NC 4.0 license (http://creativecommons.org/licenses/by-nc/4.0/). 